\title{
Crowd Behavior Mining with Virtual Environments
}

\begin{abstract}
This article explores ways in which virtual environments can be used for crowdsourcing and behavior mining for filling gaps within the information space of topical research. Behavior mining in this article refers to the act of harvesting the latent or instinctive behavior of participants, usually a crowd, and injecting the population behavior into a preset context, such as within a virtual environment so that the subjective behaviors and the contexts are merged. The experimental approach combines various modalities centered upon virtual environments so as to induce presence in order to bring participants into the context. This approach is new and not well studied; however, it has real potential in research dealing with behaviors and culture in reconstructed virtual environments. Two virtual environments case studies at the 2012 and 2015 Royal Society Summer Science Exhibition are presented, which demonstrate that the unique crowdsourcing activity is able to fill gaps within the information space so that answers to research questions can be more complete. Thus, by reconstructing and replicating a lost landscape, and by injecting harvested human behavior into the context of the landscape, we may be able to gather much more information than conventional methods will allow.
\end{abstract}

\section{Crowdsourcing in Archaeology}

Digital technology has real potential in facilitating new types of public engagement that are collaborative in nature (Bonacchi, 2012). This, together with activities initiated by GLAMs (Galleries, Libraries, Archives, and Museums) that engage with various levels of society at large is a very healthy way to involve, educate, and expose the public to the significance of the active body of research that is within the arts and humanities. One of the ways in which the public can be deeply involved, not

Presence, Vol. 24, No. 4, Fall 2015, 347-358

doi: 10.1 I 62/PRES_a_00239

(C) 2016 by the Massachusetts Institute of Technology as bystanders, or as audiences in public lectures, but as active participants within the very nature of the research process is crowdsourcing.

Crowdsourcing is a distributed problem-solving model; it is not only online (Brabham, 2008) but can be offline as in requiring no Internet connections, or it can be a hybrid of an offline-online distributed problemsolving approach. The Arts and Humanities Research Council (AHRC) Connected-Communities Scheme's crowdsourcing scoping study outlines the important activities in engaging the crowd with humanities research (Dunn \& Hedges, 2012). Listed and described amongst the process facets in Dunn and Hedges' scoping study were collaborative tagging, transcribing, correcting/ modifying content, linking, recording and creating content, commenting, critical responses and stating preferences, categorizing, cataloguing, contextualizing, dereferencing, mapping, and translating. Whilst the processes listed are encompassing, new needs such as the unique process described in this article are being discovered. The asset types provided in the study are, however, more thorough.

While crowdsourcing is not new (Howe, 2006) in the humanities, and more specifically in archaeology, it is a very recent endeavor. One of the seminal publications on the topic is the Participatory Museum (Simon, 2010), written to address issues of public dissatisfaction with cultural institutions in general. The goal of the participatory techniques is, according to Simon, "to meet visi-

\section{Eugene Ch'ng*}

Big Data and Visual Analytics Lab

School of Computer Science

University of Nottingham Ningbo China

Science and Engineering Building

199 Taikang East Road

315100 Zhejiang Ningbo, China

*Correspondence to eugene.chng@nottingham.edu.cn. 
tors' expectations for active engagement and to do so in a way that furthers the mission and core values of the institution. Rather than delivering the same content to everyone, a participatory institution collects and shares diverse, personalized, and changing content co-produced with visitors. It invites visitors to respond and add to cultural artifacts, scientific evidence, and historical records on display." This changing view toward visitor engagement with museum contents will invariably attract wider audiences, more than the remaining "older and whiter" generation, and can be seen as an active museum crowdsourcing activity. The distributed activity and products are termed in Simon's article as "crowdsourced exhibit" and "crowd-curated exhibition," with contents being the center and participants getting input from, contributing to, and sharing with each other as the social aspect of crowdsourcing.

Beyond the museum environment, in actual archaeological research, GIS data manipulation can be "volunteered" (Sylaiou, Basiouka, Patias, \& Stylianidis, 2013) by crowdsourcing on archaeological site detection, historical maps dereferencing and rectification, combating illicit trafficking of antiquities, using the information to create 3D Web GIS, solving archaeological research questions, and recording of archaeological sites by volunteers. The item "archaeological research questions solving" references a work by Masinton (2011), which investigated the use of space at medieval Bodiam castle for predicting how the space would organize the participants. The GeoExposures (Powell, Nash, \& Bell, 2013) crowdsourcing website developed by the British Geological Survey (BGS) uses the Ushahidi Crowdmap service for recording temporary geological exposures in Great Britain that might be lost to science. Arts and humanities research, particularly in archaeology, has witnessed a surge of crowdsourcing and citizen science efforts. Indeed, "the rapid pace of change within Internet technologies has significantly expanded potential for this 'digital' form of public archaeology practise"

(Richardson, 2013).

Apart from the few prominent examples given, crowdsourcing is at its infancy in the discipline. Neither in archaeology nor in other fields do we see evidence of works related to crowd behavior mining.
Crowdsourcing takes advantage of the wisdom of the crowds (Surowiecki, 2005), where the capacity of the collective intelligence, the "universally distributed intelligence," solves real-world problems (Levy, 1997). However, it is important to note that the majority of present crowdsourcing projects in archaeology specifically, and in the humanities more generally, have been a small group of highly active contributors doing the majority of the work, a case of the Pareto principles where $20 \%$ contribute to $80 \%$ of the work. With crowd behavior mining, participation can be more evenly distributed, and thus, a genuine wisdom of the crowds is sought.

Empirical studies have shown that patterns generated by collaborative tagging are extremely stable (Golder \& Huberman, 2006), and that crowd-sourced tags may be quite different from curator-defined tags (Trant, 2009). While these examples are very specific strands of work, they highlight the need to distribute effort and intelligence to the crowds, and perhaps even as a means of harvesting their behaviors. As Levy stated, since "no one knows everything, everyone knows something, [and] all knowledge resides in humanity" (Levy, 1997), and therefore harvesting the wisdom of the crowds for solving difficult problems is an important process in 21 stcentury technology-enthused research processes. Rather than letting any individual experts dictate information, wisdom can be found in the crowds.

\section{Behavior Mining with Crowds within Virtual Environments}

Crowdsourcing is new in archaeology, and although the works that are being done are seminal and exemplary, they are few and far between. For the most part, crowdsourcing within the humanities segments large tasks into sub-tasks, which participants can help to solve rather than distributing the tasks among parallel computer algorithms. Other crowdsourcing work takes advantage of the motor skills and intelligence of crowds as a collective contribution. There has not been any work so far that involves harvesting latent or instinctive behavior from the crowds in order to help answer humanities research questions. It is probable that there has never 
been such a need, until complex archaeological projects with large spatial-temporal scales such as the Europe's Lost World project and the Stonehenge Hidden Landscapes project came on the scene (Ch'ng et al., 2011; Ch'ng \& Stone, 2006; Gaffney et al., 2012; Gaffney, Fitch, \& Smith, 2009). The scale of these interdisciplinary projects pushes boundaries beyond that of conventional methods, and therefore, new ways of thinking become necessary. The case studies in this article are based on the two aforementioned research projects.

Virtual environments research where crowd intelligence is being harvested has not been looked into; this is particularly true with what this article terms "behavior mining." The term used here is not the same as mining behavior from data, also termed by a tiny collection of articles as "behavior mining" within the data-mining sub-disciplines, i.e., linking semantics with textual data.

"Behavior mining" in this article refers to the act of harvesting the latent behavior or instinctive behavior of participants, usually a crowd, and injecting the population behavior into a preset context, such as within an environment so that the subjective behaviors and the context are merged. This is a novel approach and has real potential in research dealing with cultures. This unique crowdsourcing activity may be able to fill gaps within the information space so that answers to research questions can be more complete. Thus, by reconstructing and replicating a lost landscape, and by injecting harvested human behavior into the context of the landscape, we may be able to gather much more information than conventional methods will allow (e.g., Ch'ng, Gaffney, \& Hakvoort, 2014). Within such works, the collection of a sufficiently large sample of behaviors will reveal to us important trends and patterns as human activities increase.

\section{Crowd Behavior Mining in Public Spaces}

Two case studies of crowd behavior mining are presented here. The first is part of the Europe's Lost World project and the second is the $€ 8$ million European-funded Stonehenge Hidden Landscapes project (Ch'ng et al., 2011; Gaffney et al., 2012). Both have been well funded, which shows their importance to Europe's strategic plans in culture and heritage. The for- mer has recently been awarded the prestigious $€ 2.5$ million Lost Frontiers European Advanced Research Grant, which explores inundated palaeolandscapes in the southern North Sea by using cutting-edge technologies. One of the computational and visualization strands is complex systems modeling which the author leads.

Both research studies were selected for exhibition at the prestigious Royal Society Summer Science Exhibition-Drowned Landscapes: Europe's Lost World (RS-DL, 2012) and Stonehenge Underground (RS-DL, 2012 ). The selection rate was $\sim 4 \%$ (22 exhibits out of 550 proposals for 2015). The virtual environments used for behavior mining for both exhibitions were developed using the Unity3D Integrated Development Environment (IDE) and displayed via our multitouch Mechdyne custom-made 40 -inch tabletop computer and the 40-inch Samsung SMSUR40, respectively. Both displays were made the centerpiece of the exhibit and therefore attracted wide participation, resulting in the collection of a large sample $(n>300)$ of crowd behaviors.

No evaluation of the state of presence on participants was conducted as it was a public, "in the wild" crowdsourcing exercise. However, the behavioral data captured during the exhibition were consistent with human behaviors in the past. The Drowned Landscapes data has been published (Ch'ng et al., 2014), and the Stonehenge Landscape data are being analyzed at the time of writing. Both datasets demonstrated trends and patterns that are significant, and are useful for testing hypotheses in our research.

\section{I Gaps Within the Information Space}

The need for behavior mining as a crowdsourcing activity within virtual environments is attributed to the gaps in the information space (see Figure 1 ) of the two strands of research described earlier. In landscape archaeology, both accessible and submerged archaeological landscapes contain natural data (see Figure 1.1), which are at best fragmentary as the intangible cultural and behavioral aspects, and very likely the tangible aspects associated with the former might have been lost, inaccessible, or disintegrated due to environmental factors (i.e., cultures moved on, leaving only sparse traces of human ac- 


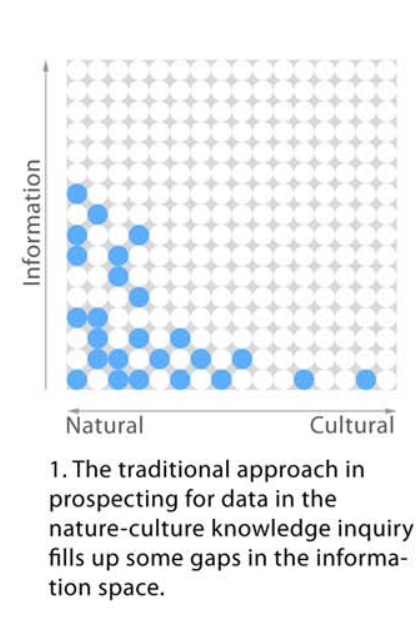

Figure I. Incremental approaches filling up the information space.

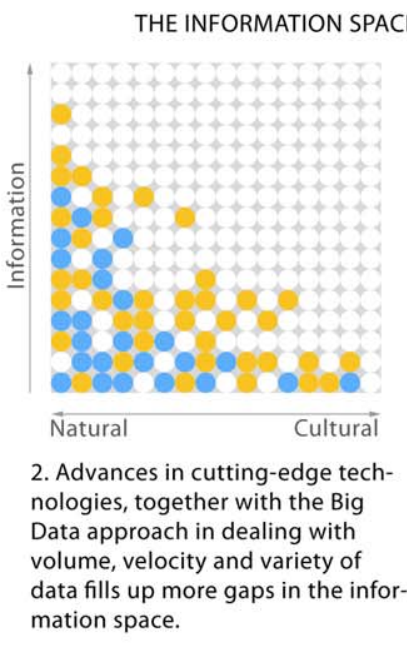

data fills up more gaps in the information space. tivity). Marine coring may be able to acquire biological samples via pollen and sedaDNA (Smith et al., 2015), environmental records could be reliably determined to within a local region, and high-resolution geomorphological changes may be accurately captured using remote sensing devices. Data analytics of processed datasets, together with the development of cutting-edge technologies, and subject-matter methodology within archaeology could infer and fill up more of the information space (see Figure 1.2). However, the fact is, a large amount of population activity, spanning thousands of years, would have been lost.

Advances in agent-based modeling (see Figure 1.4) as applied in palaeoenvironments may be able to solve such problems in the future (Ch'ng \& Gaffney, 2013; Ch'ng, 2009; Ch'ng, 2013), but human behavior mining (see Figure 1.3) can be a complementary approach that requires limited developmental resources, is quick, and diversity is implied via larger demographics group sampling.

\subsection{Drowned Landscapes Virtual Environment}

The crowdsourced behavior mining exercise for the Drowned Landscapes exhibit (3-6 July 2012, at Carlton House Terrace, London) has been published with the title "Stigmergy in Comparative Settlement
Choice and Palaeoenvironment Simulation" (Ch'ng et al., 2014). Global warming at the end of the last Ice Age led to the inundation of vast landscapes in the southern North Sea that were once inhabited by thousands of people. The landscapes, which represent large submerged fragments distributed across the world are now inaccessible; however, seismic reflectance data have provided a detailed map of the prehistoric topography (Gaffney et al., 2009). A theoretical landscape that incorporates models of real-world data from previous studies (Ch'ng, Stone, \& Arvanitis, 2004; Ch'ng \& Stone, 2006) were created for the crowdsourcing exercise (see Figure 2).

The crowd behavior mining activity probed questions related to how individuals would respond to a changing palaeoenvironmental setting, where participants have one opportunity to build a settlement on a coastal landscape, balancing safety and access to resources, including sea and terrestrial foodstuffs, while taking into consideration the threat of rising sea levels in the face of climate change and coastal inundation. The activity also probes the question of stigmergy (Grassé, 1959, 1984)—cooperation without direct communication. The results of the study considered whether decisions on settlement were predicated to be near to locations where previous structures were built, and whether later settler choice would fare better, and score higher 


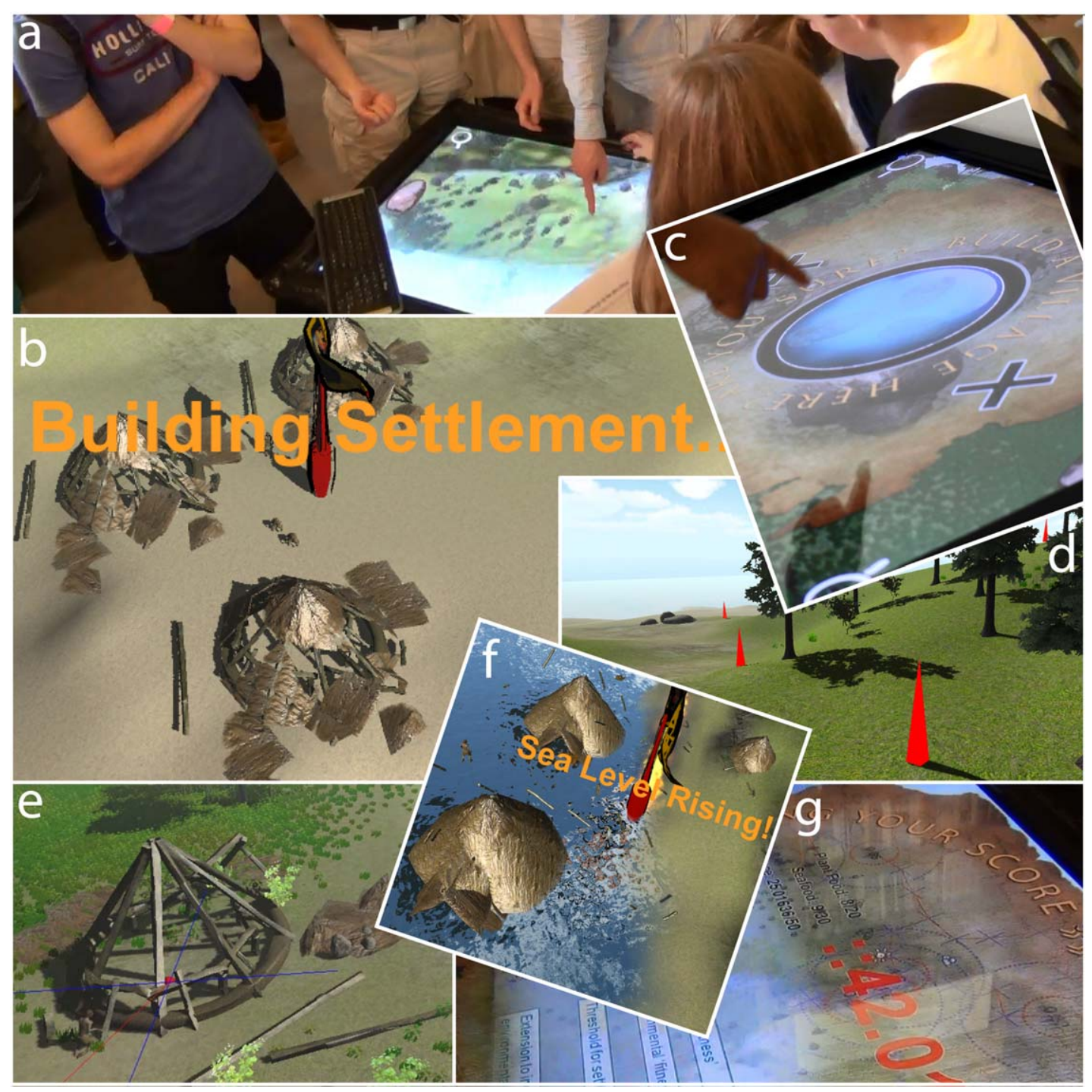

Figure 2. Submerged Landscapes —Europe's Lost World crowd behavior mining simulator. (a) Participants interacting with the simulation. (b) Mesolithic virtual agents building a settlement. (c) Participant confirming the building of a settlement. (d) Red-colored 3D icons in the landscape showing settlement locations of previous participants (stigmergy). (e) Virtual agents building a mesolithic house. (f) Sea level rising and destroying the settlement. (g) Scores calculated from the simulation, weighing between safety from sea level rises and resources.

as time progressed, having "learned" from earlier settlers.

A total of 347 participant behaviors were harvested, leaving a trail of patterns on the virtual landscape. Data analysis demonstrated that participant preferences follow a certain priority, such as the large river mouth, sparse woodlands near coastal areas, and the lake. Settlers kept away from islands, open areas including grasslands and coasts, ravines, treacherous coastal areas, and rocky bays. Evidence also showed that participants worked via the principles of stigmergy, by locating existing markers to identify best locations to build upon even though there were alternate locations, which might also be good sites. In short, participants learned from past behaviors. This is a very good case of 
crowd behavior mining, which prompted the second activity, discussed in the next section.

\subsection{Stonehenge Underground Virtual Environment}

The crowdsourced behavior mining exercise for the Stonehenge Hidden Landscapes project has gathered 342 participant behaviors during the Royal Society Summer Science Exhibition (30 June-5 July 2015, at Carlton House Terrace, London) and the results are being analyzed at the time of writing.

Stonehenge is a circular stone monument constructed and posted within England's Salisbury Plain. The landscape has 9,000 years of human activities centered on and around the stones. Cutting-edge remote sensing techniques and geophysical surveys, the largest of its kind, have revealed hundreds of new features that now form part of the most detailed archaeological digital map of the Stonehenge landscapes ever produced (Gaffney et al., 2012). The survey also revealed 17 previously unknown ritual monuments dating to the period when Stonehenge achieved its iconic shape. Evidence suggests that the landscape is a large burial ground with huge religious importance in its time. The project has brought together interdisciplinary expertise in noninvasive techniques to address gaps in our knowledge and understanding of the landscape context.

The topology of the Stonehenge landscape is processed via software developed within the project, manipulated in GIS, and finally ported into the Unity3D IDE before the user interface was integrated with the virtual environment (see Figure 3). The topology was exaggerated and texture mapped to bring out contour information. Original barrows were placed on their respective locations together with 3D models of the larger monuments.

The crowd behavior mining activity probes questions related to location choices of burial mounds in relation to prominent monuments such as Stonehenge, the Cursus (a 3-km monument), the Blue Henge down by the river Avon, as well as the complex landscape topology. Gender biases, age, and choice of grave goods selected by participants were composed of amber necklaces, bronze daggers, jet necklaces, gold bracelets, ceremonial mace, and beaker pottery. Six choices of burial mounds were also given: bowl barrow, bell barrow, bell barrow with bank, disc barrow, pond barrow, and saucer barrow. Each choice of grave goods has a historical significance of use, and they can be ranked in terms of the importance of the person being buried. The size and height of the barrows affect visibility from and toward Stonehenge, together with the choice of location, and the prominence of the burial site will be known after analysis. The activity also probes the question of stigmergy, as in how initial burial sites will affect latter ones, that is, how ancient people separated by hundreds of years can actually cooperate without direct communication, and whether clustering will occur between similar genders, grave goods, and barrow types. Presently, crowd behavior data are being analyzed.

\section{Issues on Crowdsourcing with Virtual Environments}

There are, however, issues with using virtual environments for behavior mining, as far as presence is concerned. Behavior mining requires participants to be placed within the context of the subject-specific environment, and presence is a mandatory component. One of the issues is our ability to induce presence in virtual environments, especially when the display is within a public space, such as during the Royal Society Summer Science Exhibition.

Here, it will be useful to bring up the definition of presence again for the sake of readership, as well as cover some of the related theoretical and empirical works that we can apply in our crowdsourcing work.

Presence is defined by Slater and Wilbur (1997) as "a state of consciousness, the [psychological] sense of being in the virtual environment." Presence is the "perceptual illusion of non-mediation" (Lombard \& Ditton, 1997) with six different conceptualizations: presence as social richness, presence as realism, presence as transport, presence as immersion, presence as social actor within medium, and presence as medium as social actor.

One of the first issues with crowdsourcing using virtual environments is the use of appropriate devices as our 


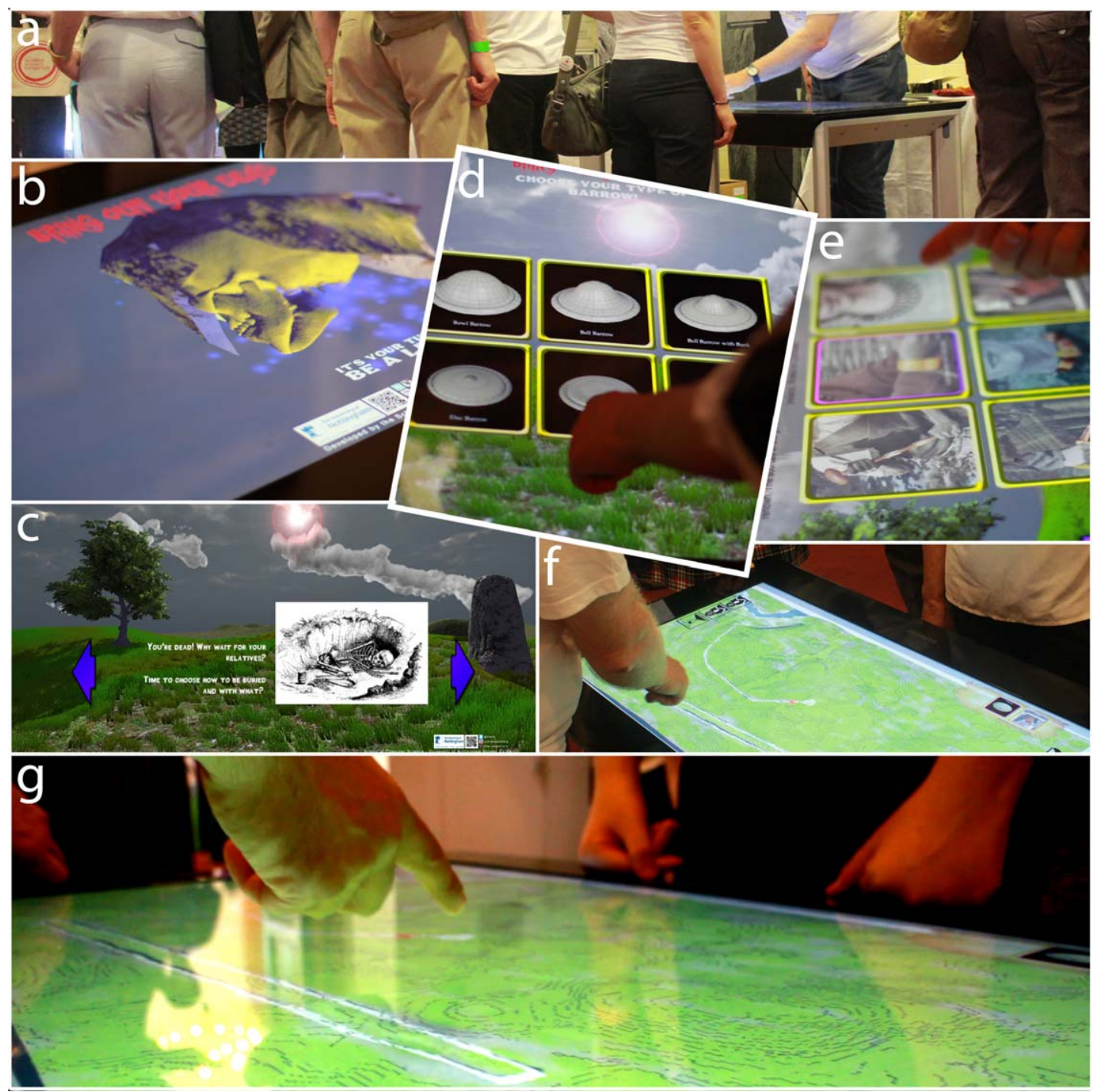

Figure 3. Stonehenge Underground - Stonehenge Hidden Landscapes crowd behavior mining simulator. (a) A crowd viewing the exhibit and participating in the behavior mining exercise. (b) The "skull" introduction screen attracting attention. (c) One of the narrative screens. (d) Participant selecting the barrow type. (e) Participant selecting a grave good. ( $f$ and $g$ ) The map view with overlapping contours.

medium. Certain strands of work require mobile devices of different screen sizes, that is, phones, tablets, phablets. Others can be online Web contents presented as a page with embedded $3 \mathrm{D}$ virtual environments. These approaches can be left entirely to the users as long as the instructions are clear. Others require a more personal touch, such as the case studies presented in this article. However, each has its own limitations. With mobile phones or Web 3D environments, users are left on their own without guidance. Furthermore, screen sizes will be irregular, and presence in small displays is not well studied. Public displays that have larger screen sizes may be appropriate; however, distractions are a hindrance as the exhibits are in a public space, with potentially continuous streams of visitors walking around. There is, therefore, no sensory suppression of the immediate environment (Biocca, 1999) as it is a display at the center of the exhibit. 
The case studies presented in this article have important stories and information that require a narrative in the form of a storytelling. The Royal Society, too, requires that we guide our visitors into the content of our exhibits. As a result, our highly enthusiastic archaeology professors, who all are great storytellers, were there all day for the narratives. Therefore, a large display in the format of a tabletop computer with multitouch capability was our choice.

A tabletop format is indeed a good choice for exhibits. Tabletops can be a familiar setting where users crowd around in a collaborative manner either working together, or under the guidance of a leader or teacher. It can be hypothesized that information can be disseminated more effectively due to the familiar and "enclosed" setting-users can gather in a semi-circle forming a private space, much like storytelling in the past. A good narrative accompanying a display can "transport" a user into the story and therefore, self-presence (Biocca, 1999) is induced to a certain extent, as the user sees him or her "self" as within the environment during the activity.

It is well known that psychological immersion can be induced via mediation, such as verbal, or even written contents. For example, it is known that reading novels or written narratives could transport a reader into the story (Gerrig, 1993). Verbal narratives are a catalyst for mental immersion. Narratives are stories that users can inhabit from a first-person perspective where they are engaged in the experience of mediated presence (Gorini, Capideville, De Leo, Mantovani, \& Riva, 2011; Sherman \& Craig, 2003). Narratives of objects and environments can induce mental pictures in the minds of their readers (Bransford, Barclay, \& Franks, 1972). Furthermore, it was demonstrated that emotional contents conveyed via narratives are attributed to generating emotional response, and strengthening the subjects' sense of inner presence in mediated space (Gorini et al., 2011). A good narrative in the form of storytelling accompanying a virtual environment can greatly augment a sense of presence for users.

Virtual environments must also convey a sense of place. There is a need to put individuals of the crowd into the "place." A sense of place is important in the understanding and familiarity of a landscape. "Places are sensed in a chiaroscuro of setting, landscape, ritual, routine, other people, personal experience, care and concern for home and the context of other places" (Relph, 1976). Tuan (1977) proceeds to describe place as one which can increase with value as more information is received: "What begins as undifferentiated space becomes place as we get to know it better and endow it with value." How then does one create a sense of place when participants only spent at most $\sim 30$ minutes exploring the virtual environment? The solution may be to provide a richer set of knowledge than if participants were to acquire an experience of the landscape, without having the time to physically explore it. As stated earlier, this can be achieved in a number of ways with the increase of information.

\section{I Use Narratives to Augment Contextual Information}

First, narratives are important. It makes a difference when a very personalized narrative accompanies participants. The storytelling that was given was interactive. In the case of the Stonehenge Underground display, questions were asked of participants and by participants during the $\sim 20$-minute session so that they might become more engaged in conversations with our leading archaeologists. This resulted in a greater depth of understanding of the subject context. This invariably increases the perceived value of the "place." Knowledge of archaeologists can be transferred via narratives and, as a result, participants become familiar with the landscape context very quickly. Studies have also shown that people can construct accurate spatial representations of environments via verbal descriptions (Ehrlich \& Johnson-Laird, 1982; Mani \& Johnson-Laird, 1982), in one, two and three dimensions (Foos, 1980; Franklin \& Tversky, 1990).

\subsection{Design User Interfaces for Crowdsourcing}

The second factor that augments information is the medium-a multitouch tabletop computer as the user interface device. Participants would have been very 
familiar with touch-based interfaces, mainly through the accessibility of smartphones and tablets. Due to prior experience with touch displays, albeit smaller-sized screens, we found that users were able to quickly learn to navigate the virtual environments, usually within $\sim 1$ minute of first use. The horizontal interface too allows a larger portion of the virtual landscape to be explored in a much shorter time than a conventional "first-person" experience of a virtual environment. This invariably decreases the required length of participation time and thus increases the opportunity for data sampling. We designed the user interfaces of the case studies to be simple and intuitive, with direct access to simple features (zoom in and out, bury, etc.). In both cases, user evaluation was not necessary, as our participants of all ages familiarized themselves with the user interfaces rather quickly.

A rich set of information was also provided within the display to augment the contextual information. The topology of the virtual landscape, for example, was made more prominent without distorting the landscape. Contour lines can be switched on/off to show more of the landscape elevation model. The Stonehenge Underground display had two viewing options: map view and first-person view helps users orientate themselves within the landscape, both with zoom in/out functions. The map viewing option was not necessary for the Drowned Landscapes virtual environment because, unlike Stonehenge Underground, the fine detail of the topology was not an important scoring factor. All these were designed to assist users in creating a mental model of the virtual space. Together with the narratives, we found that users were able to confidently contribute to our crowdsourcing exercise.

A short 20-minute session is sufficient to put our participants into the context of the landscape with the augmented information described previously. This is justifiable as, in the case of Stonehenge, ancient inhabitants of the landscape travelled from afar to bury their dead and may not have known the landscape well. They were likely to have been introduced to the landscape, or, have heard stories of the landscape well enough to choose a burial site, all within a relatively short period of time. In the case of Doggerland, hunter-gatherers roamed the landscapes and settled in order to survive, without necessarily having thoroughly explored the landscape. Both are typical of peoples of the land at their respective time thousands of years ago.

\subsection{Utilize Special Effects for Harvesting Behaviors}

Third, there are challenges with separating modern intellectual behavior from latent or instinctive behaviors. Although this is not absolutely critical to our research presented here, it may become necessary for future behavior mining activities. This can be induced via various effects, such as the urgency of time by adding a "panic" soundtrack with a countdown timer as warning, together with the narratives. These effects were used in the Submerged Landscapes virtual environment for harvesting the necessary instinctive behaviors (Ch'ng et al., 2014).

\section{Challenges in Mining Crowd Behavior}

This article describes the difficulty, open questions, and challenges associated with crowdsourcing using virtual environments through the presentation of two case studies at the Royal Society Summer Science Exhibition 2012 and 2015. The novel approach described here, in which behaviors could be mined using virtual environments, is the result of large-scale research projects. These projects require new ways of thinking so that gaps in the information space could be filled. The two case studies presented here are but the beginning of a series of studies requiring the application of virtual environments in the arts and humanities. Perhaps such ideas may also be applied to the sciences that involve human behaviors. Cutting-edge technologies such as those developed and applied within the case studies have acquired large-scale datasets never before studied in our individual disciplines. The volume of such data spans both space and time, and the variety thereof means that a rethink of our methodology has become necessary. The experiments with behavior mining were borne out of that necessity, and have proven to be useful. However, harvesting 
human behavior in the crowdsourcing context has many open problems, and this article is but a starting point.

A greater need to study crowdsourcing using virtual environments is necessary. Presented in the following list are key challenges in using virtual environments for crowd behavior mining. These problems may somewhat overlap but they are not wholly independent of each other.

- Presence is an important component in crowd behavior mining. The presence felt while using virtual environments does affect participant behavior. How do we measure presence, or conduct evaluation using virtual environments in both public and private spaces?

- How can presence be induced in public exhibitions; conversely, how can presence be induced within more private spaces such as on mobile or Web virtual environments?

- Narratives play an important role in public exhibits. Visitors are always keen to hear a good story; however, there is a limit to dedicated human resources for such tasks. In order to achieve the same effect, which modalities or combinations of modalities are more effective?

- Behavior mining in the crowdsourcing context requiring large samples will need to be distributed on mobile devices or the Web so that data collection is maximized. Which modalities or combinations of modalities are able to achieve the same effect on different display devices?

- Visitors come in groups. There is usually a single participant, surrounded by nonparticipants; however, groups interact as a whole and frequently decisions made are normalized, and become an average behavior of the group. This does not present a problem in the two case studies, as ancient behaviors associated with the context were collective-they socialize as a group. This may present problems for topic-specific projects where group noise is unwelcomed.

- Artificial agents are potential tools in distributed problem-solving models. In the case where specific behaviors will need to be homogenized via artificial agents, how can artificial agents complement crowd behavior within virtual environments?

The list of challenges is both generic and specific, and could be extended, of course. It represents groundwork for 21 st-century research involving the connected public as stakeholders, and an initial exploration into the need for crowd-mining behavior in order to complement other nonhuman datasets. Behavior mining is an initial step toward the understanding of discipline-specific research questions, but it could well be research on its own, especially within the virtual environments community.

\section{Acknowledgments}

The author wishes to thank the Royal Society for their tremendous support for the 2012 and 2015 Royal Society Summer Science Exhibition, which made this new strand of research possible. The author also wishes to express gratitude for the School of Computer Science's support, from both the University of Nottingham's UK and China campuses.

\section{References}

Biocca, F. (1999). The cyborg's dilemma: Progressive embodiment in virtual environments. Human Factors in Information Technology, 13, 113-144.

Bonacchi, C. (2012). Archaeology and digital communication. Towards strategies of public engagement. London: Archetype Publications.

Brabham, D. C. (2008). Crowdsourcing as a model for problem solving: An introduction and cases. Convergence: The International Journal of Research into New Media Technologies, 14(1), 75-90.

Bransford, J. D., Barclay, J. R., \& Franks, J. J. (1972). Sentence memory: A constructive versus interpretive approach. Cognitive Psychology, 3(2), 193-209.

Ch'ng, E. (2009). An artificial life-based vegetation modelling approach for biodiversity research. In R. Chiong (Ed.), Nature-inspired informatics for intelligent applications and knowledge discovery: Implications in business, science and engineering (pp. 68-118). Hershey, PA.: IGI Global.

Ch'ng, E. (2013). Model resolution in complex systems simulation: Agent preferences, behavior, dynamics and n-tiered networks. SIMULATION, 89(5), 635-659. 
Ch'ng, E., Chapman, H., Gaffney, C., Gaffney, V., Murgatroyd, P., \& Neubauer, W. (2011). From sites to landscapes: How computing technology is shaping archaeological practice. IEEE Computer: Special Issue on Computational Archaeology, 44(7), 40-46.

Ch'ng, E., Gaffney, V., \& Hakvoort, G. (2014). Stigmergy in comparative settlement choice and palaeoenvironment simulation. Complexity, 21: 59-73. doi: 10.1002/cplx.21616

Ch'ng, E., \& Gaffney, V. L. (2013). Simulation and visualisation of agent survival and settlement behaviours in the hunter-gatherer colonisation of Mesolithic landscapes. In E. Ch'ng, V. L. Gaffney, \& H. Chapman (Eds.), Visual heritage in the digital age (pp. 235-258). Springer Cultural Computing Series.

Ch'ng, E., \& Stone, R. J. (2006). Enhancing virtual reality with artificial life: Reconstructing a flooded European Mesolithic landscape. Presence: Teleoperators and Virtual Environments, 15(3), 341-352.

Ch'ng, E., Stone, R. J., \& Arvanitis, T. N. (2004). The Shotton River and Mesolithic dwellings: Recreating the past from geo-seismic data sources. In K. Cain, Y. Chrysanthou, F. Niccolucci, \& N. A. Silberman (Eds.), The 5 th international symposium on virtual reality, archaeology and cultural heritage, VAST04: Interdisciplinarity or "the best of both worlds": The grand challenge for cultural heritage informatics in the 21st century (pp. 125-134). Brussels, Belgium: Citeseer.

Dunn, S., \& Hedges, M. (2012). Crowd-sourcing in the humanities: A scoping study. Engaging the crowd with humanities research. UK Arts and Humanities Research Council Connected Communities Scheme. Retrieved from http://crowds.cerch.kcl.ac.uk/wp-content/ uploads/2012/12/Crowdsourcing-connected -communities.pdf

Ehrlich, K., \& Johnson-Laird, P. N. (1982). Spatial descriptions and referential continuity. Journal of Verbal Learning and Verbal Behavior, 21(3), 296-306.

Foos, P. W. (1980). Constructing cognitive maps from sentences. Journal of Experimental Psychology: Human Learning and Memory, 6(1), 25.

Franklin, N., \& Tversky, B. (1990). Searching imagined environments. Journal of Experimental Psychology: General, $119(1), 63$.

Gaffney, C., Gaffney, V., Neubauer, W., Baldwin, E., Chapman, H., Garwood, P., . . Löcker, K. (2012). The Stonehenge hidden landscapes project. Archaeological Prospection, 19(2), 147-155.
Gaffney, V. L., Fitch, S., \& Smith, D. (2009). Europe's lost world: The rediscovery of Doggerland. CBA Research Report. No. 160.

Gerrig, R. J. (1993). Experiencing narrative worlds. New Haven, CT: Yale University Press.

Golder, S. A., \& Huberman, B. A. (2006). Usage patterns of collaborative tagging systems. Journal of Information Science, 32(2), 198-208.

Gorini, A., Capideville, C. S., De Leo, G., Mantovani, F., \& Riva, G. (2011). The role of immersion and narrative in mediated presence: The virtual hospital experience. Cyberpsychology, Behavior, and Social Networking, 14(3), 99-105.

Grassé, P. P. (1959). La théorie de la stigmergie: Essai d'interprétation du comportement des termites constructeurs. Insectes Sociaux, 6, 41-81.

Grassé, P. P. (1984). Termitologia, Fondation des Sociétés. Construction. Tome II. Paris, Masson.

Howe, J. (2006). The rise of crowdsourcing. Retrieved from http://www.wired.com/wired/archive/14.06/crowds.html

Levy, P. (1997). Collective intelligence: Mankind's emerging world in cyberspace. Cambridge, MA: Perseus Books.

Lombard, M., \& Ditton, T. (1997). At the heart of it all: The concept of presence. Journal of Computer-Mediated Communication, 3(2), 0.

Mani, K., \& Johnson-Laird, P. N. (1982). The mental representation of spatial descriptions. Memory \& Cognition, 10(2), 181-187.

Masinton, A. (2011). Human guinea pigs and casual collaborators: Crowdsourcing archaeological data. CAA 2012: The Computer Applications and Quantitative Methods in Archaeology Annual Conference.

Powell, J., Nash, G., \& Bell, P. (2013). GeoExposures: Documenting temporary geological exposures in Great Britain through a citizen-science web site. Proceedings of the Geologists' Association, 124(4), 638-647.

Relph, E. (1976). Place and placelessness (Vol. 67). London: Pion.

Richardson, L. (2013). A digital public archaeology? Papers from the Institute of Archaeology, 23(1), Art-10.

RS-DL. (2012). Drowned landscapes: Europe's lost world. Retrieved from http://sse.royalsociety.org/2012/exhibits/ drowned-landscapes/

Sherman, W. R., \& Craig, A. B. (2003). Understanding virtual reality: Interface, application, and design. San Francisco, CA: Morgan Kaufmann.

Simon, N. (2010). The participatory museum. Museum 2.0. 
Slater, M., \& Wilbur, S. (1997). A framework for immersive virtual environments (FIVE): Speculations on the role of presence in virtual environments. Presence: Teleoperators and Virtual Environments, 6, 603-616.

Smith, O., Momber, G., Bates, R., Garwood, P., Fitch, S., Pallen, M., ... Allaby, R. G. (2015). Sedimentary DNA from a submerged site reveals wheat in the British Isles 8000 years ago. Science, 347(6225), 998-1001.

Surowiecki, J. (2005). The wisdom of crowds: Why the many are smarter than the few. London: Abacus.
Sylaiou, S., Basiouka, S., Patias, P., \& Stylianidis, E. (2013). The volunteered geographic information in archaeology. ISPRS Annals of the Photogrammetry, Remote Sensing and Spatial Information Sciences, 301-306.

Trant, J. (2009). Tagging, folksonomy and art museums: Results of steve.museum's research. Technical Report, Archives and Museum Informatics.

Tuan, Y. F. (1977). Space and place. Minneapolis: University of Minnesota Press. 